\title{
A Mobile Agent Team Works based on Load-Balancing Middleware for Distributed Computing Systems
}

\author{
Fatéma Zahra Benchara ${ }^{1}$, Mohamed Youssfi ${ }^{2}$ \\ Department of Computer Science, Laboratory SSDIA \\ ENSET Mohammedia, Hassan II University of Casablanca, Mohammedia, Morocco
}

\begin{abstract}
The aim of this paper is to present a load balancing middleware for parallel and distributed systems. The great challenge is to balance the tasks between heterogeneous distributed nodes for parallel and distributed computing models based distributed systems, by the way to ensure HPC (High performance computing) of these models. Accordingly, the proposed middleware is based on mobile agent team work which implements an efficient method with two strategies: (i) Load balancing Strategy that determines the node tasks assignment based on node performance, and (ii) Rebalancing Strategy that detects the unbalanced nodes and enables tasks migration. The paper focuses on the proposed middleware and its cooperative mobile agent team work model strategies to dynamically balance the nodes, and scale up distributed computing systems. Indeed, some experimental results that highlight the performance and efficiency of the proposed middleware are presented.
\end{abstract}

Keywords-Load balancing; middleware; parallel and distributed systems; parallel and distributed computing models; high performance computing; mobile agents; distributed computing

\section{INTRODUCTION}

Distributed systems play a great role by providing a promising distributed computing environment for big data applications, in order to meet their requirements, and ensure the HPC. Therefore, distributed systems have been introduced as a promising solution for HPC thanks to two main features: interconnection network speed such as: Ethernet, 4G, 5G and, their effective Middleware such as: CORBA, RMI, AMQP. These make distributed systems as a cooperative parallel and distributed environment able to implement parallel and distributed computing models, and ensure the collaboration of heterogeneous machines in order to achieve the processing power required by big data [1] applications, and reduce the computing time.

Consider the two principal distributed system challenges that these applications have to deal with. Their scalability and efficiency depends on their ability to manage the message passing paradigm, and the heterogeneity of node performance. For example, performing an application of big data classification based parallel and distributed computing models. It involves a wide number of heterogeneous distributed computing system nodes to achieve the required processing power. The heterogeneity of distributed system nodes influences negatively the performance of these models if unbalanced task assignment is performed between nodes.
Therefore, an effective task assignment strategy is required to deal with the load balancing challenge.

In this paper, a new load balancing middleware is proposed, which is based on mobile agents, and implements effective method for task assignment and migration. Besides, the proposed middleware integrates a cooperative mobile agent team work model, which elaborates well defined load balancing strategies to balance the distributed computing system. Consider the great challenge of nodes performance heterogeneity in parallel and distributed systems. We will present a load balancing model that achieves these requirements. This paper is organized as follows:

- The middleware and its innovative components for load balancing process are presented in Section 3.

- The Section 4 is focused on presenting the method used by the mobile agent team work in order to elaborate load balancing strategies.

- The efficiency of proposed load balancing middleware is demonstrated, by performing an SPMD application in parallel and distributed computing system (Section 5).

\section{BACKGROUND}

To set the scene of this paper, we begin with a brief overview of distributed computing systems [2], and their ability to perform HPC application based on parallel and distributed computing models. Consider this application is composed by a set of NT tasks $T_{k}\{k=1, \ldots, N T\}$, which is executed in parallel and distributed computing system of $n$ nodes $N_{i}\{i=1, \ldots, n\}$. In case of homogenous system, the same number of tasks (load LB) is assigned for each node $\mathrm{Ni}$ with $\mathrm{LB}_{\mathrm{i}}=\frac{\mathrm{NT}}{\mathrm{n}}$. Otherwise, the load LB depends on the node's performance index $\mathrm{NPI}_{\mathrm{i}}$, by means that for each node $\mathrm{Ni}$ the assigned load LBi is given by:

$\mathrm{LB}_{\mathrm{i}}=\frac{\mathrm{NT}}{\mathrm{n}} \times \mathrm{NPI}_{\mathrm{i}}$

Therefore, a load balancing method for HPC applications based distributed system is required. This method has to take into account the node performance index $\mathrm{NPI}_{i}$, and grants the same computation time for all nodes. Thus, the computation time $\Omega^{\text {MIN }}$ of node $\mathrm{N}_{\mathrm{i}}^{\text {MIN }}$ (slowest node) is equal to $\Omega^{\mathrm{MAX}}$ of node $\mathrm{N}_{\mathrm{j}}^{\mathrm{MAX}}$ (faster node) with $\mathrm{i} \neq \mathrm{j}$. 
The Mobile agents [3],[4] have impressive skills, such as asynchronous communication ability, autonomy, adaptability, and mobility. They can move from overloaded nodes to under loaded ones, and perform a balanced system. The agent's mobility can be an effective mechanism for dynamic load balancing of the system. Additionally, the mobile agents cooperate asynchronously by exchanging messages, which significantly reduces the load balancing strategy time. Further, their adaptability skill makes the proposed middleware flexible with different distributed computing systems. Thus, the mobile agents ensure effective features for scalable load balancing middleware.

\section{PRoposed LoAd BALANCING MidDleWARE}

\section{A. Middleware Overview}

The proposed middleware (Fig. 1) implements a load balancing method, which behaves when an application is deployed, and performed in the system. Once, it is deployed this method defines the initial performance index by getting the node's performance capabilities. If the metadata of task is known, the defined index is used to estimate the load assignment $\mathrm{LB}_{\mathrm{i}}$ for each node $\mathrm{N}_{\mathrm{i}}$. Else, the initial performance index will be used for running the application. For an iterative application, before the next iteration, this method decides the required load migration. Thus, the middleware can balance the node's load effectively.

\section{B. Aspect based Load-balancing Middleware}

The proposed middleware integrates the LoadBalancer aspect to the system. This aspect is based on AOP (Aspect Oriented Programming) approach [5],[6], which is useful for separating the functional aspects from the technical ones in an application, and allows to dynamically modify the program behavior. To do so, the middleware adds two aspects (behaviors) to the system, by the way that it can get metadata and provide the results needed to balance the system. These behaviors are described as follows:

- Load Assignment Aspect This aspect is performed when an application is deployed in order to get the load assignment of nodes.

- Rebalancing Aspect This aspect is executed when an application is running in order to get the required load migration, and rebalance the system before performing the next iteration.

\section{EFFECTIVE LOAD BALANCING METHOD}

The proposed middleware implements an effective load balancing method (Fig. 2) for distributed computing system. This is done according to three method's main step; Initial nodes performance Determination, Load Assignment Prediction, Load Rebalancing. This method is implemented on cooperative mobile agent team works composed by two principal agents: Team Load Balancer Agent (TLBA agent), and Team Node Performance Monitor Agents (TNPMA agents) one per node. Main steps of this method are detailed as follows:

Step 1. Initial nodes performance Determination

- Metadata $\mathrm{MDT}_{0}$ Determination

- Computing the initial performance index $\mathrm{NPI}^{\mathrm{T} 0}$

- Computing the initial load $\mathrm{LB}^{\mathrm{T} 0}$

Step 2. Load Assignment Prediction

- Metadata $\mathrm{MDT}_{\mathrm{k}}$ Determination

- Computing the Node Performance index NPI ${ }^{\mathrm{Tk}}$

- Computing the initial load $\mathrm{LB}^{\mathrm{Tk}}$

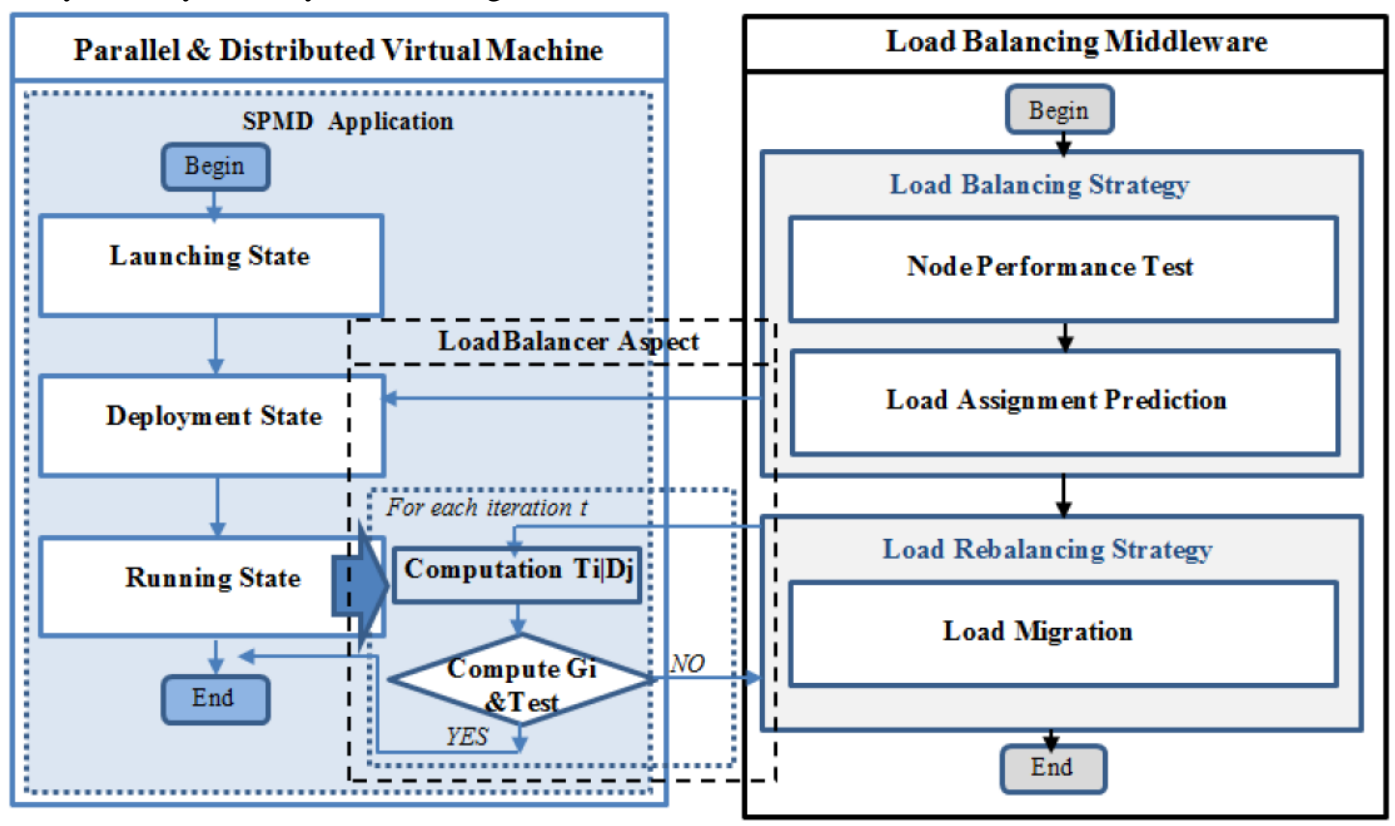

Fig. 1. Load Balancing Middleware Architecture. 


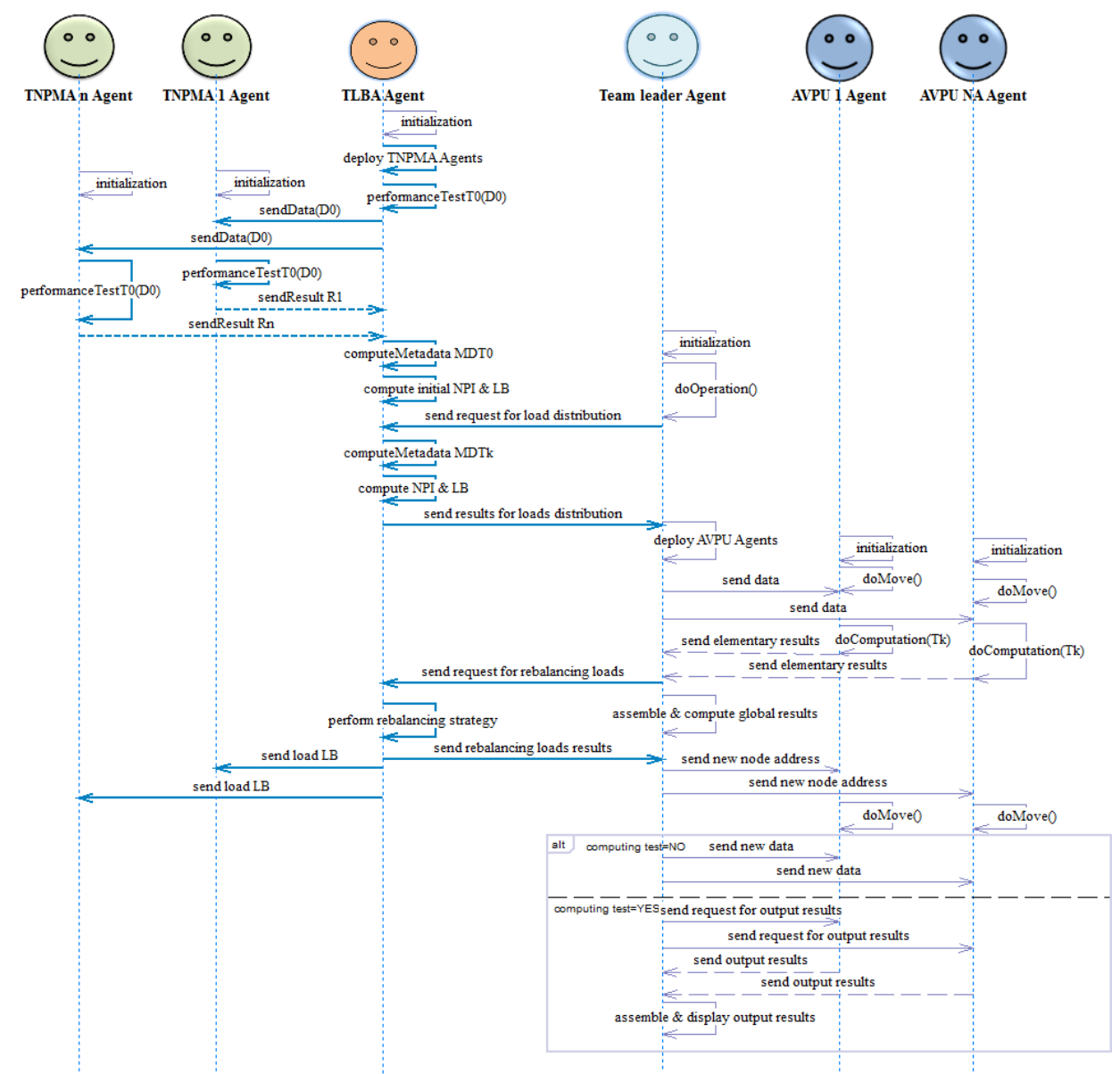

Fig. 2. Sequence Diagram of Mobile Agent Team Works Load-Balancing Method.

\section{Step 3. Load Rebalancing}

The load balancing method's steps are detailed as follows:

Step 1. Initial nodes performance Determination

\section{- Metadata $\mathrm{MDT}_{0}$ Determination}

a) The TLBA agent deploys TNPMA(i) agent for each node $\mathrm{N}_{\mathrm{i}}$, after its initialization by the task $\mathrm{T}_{0}$ (initial performance task with complexity $\mathrm{C}_{0}$ ) and the data $\mathrm{D}_{0}$ (initial data of size $\mathrm{x}_{0}$ ).

b) The TLBA agent executes the performance test of its node $\mathrm{N}_{0}$.

c) The TLBA agent sends the data $D_{0}$ for each TNPMA(i) agent.

d) Each TNPMA(i) agent gets the data $\mathrm{D}_{0}$ and executes the task $\mathrm{T}_{0}$ on data $\mathrm{D}_{0}$.

e) Each TNPMA(i) agent returns the results $\mathrm{R}_{\mathrm{i}}$ (the size of result $y_{0}$, and the execution time $\left.\theta t_{i}^{T_{0}}\right)$ to the TLBA agent.

f) The TLBA agent receives the result $\mathrm{R}_{\mathrm{i}}$ from each TNPMA(i) agent at $t_{1}(\mathrm{i})$, and computes the communication latency $\theta \mathrm{l}_{i}^{T_{0}}$ between the node $\mathrm{N}_{\mathrm{i}}$ and $\mathrm{N}_{0}$ by:

$\theta l_{i}^{T_{0}}=\omega_{i}^{T_{0}}-\theta t_{i}^{T_{0}}$
Where: $\boldsymbol{\omega}_{\boldsymbol{i}}^{\boldsymbol{T}_{\mathbf{0}}}$ computation time of the task $\mathrm{T}_{0}$ in the node $\mathrm{N}_{\mathrm{i}}$, which is given by:

$\omega_{i}^{T_{0}}=t_{1}(i)-t_{0}$

- Computing the initial performance index $\mathrm{NPI}^{\mathrm{T} 0}$

The TLBA agent computes the initial performance $\mathrm{NPI}_{i}^{T_{0}}$ of each node $\mathrm{N}_{\mathrm{i}}$ by:

$N P I_{i}^{T_{0}}=\frac{M I N\left(\omega_{i}^{T_{0}}\right)}{\omega_{i}^{T_{0}}}$

- Computing the initial load $\mathrm{LB}^{\mathrm{T} 0}$

The TLBA agent determines the initial load $\mathrm{LB}^{\mathrm{T} 0}$ of each node $\mathrm{N}_{\mathrm{i}}$ by:

$L B_{i}^{T_{0}}=\mathrm{LB}_{\mathrm{ref}} \times \frac{N P I_{i}^{T_{0}}}{\left\langle N P I_{i}^{T_{0}}\right\rangle}$

Where:

$\mathrm{LB}_{\text {ref }}$ The referenced load of each node $\mathrm{N}_{\mathrm{i}}$ in homogeneous distributed system, which is computed by (6), where NT is the total number of tasks, and $\mathrm{n}$ the total number of nodes.

$\mathrm{LB}_{\mathrm{ref}}=\frac{N T}{n}$ 
$\left\langle\boldsymbol{N P I _ { i }}{ }^{\boldsymbol{T}_{\mathbf{0}}}\right\rangle$ The average of node performance index $\mathrm{NPI}_{\mathrm{i}}$ that is computed by.

$\left\langle N P I_{i}^{T_{0}}\right\rangle=\frac{\sum_{i=0}^{n-1} N P I_{i}^{T_{0}}}{n}$

\section{Step 2. Load Assignment Prediction}

- Metadata $\mathrm{MDT}_{\mathrm{k}}$ Determination

The TLBA agent predicts the execution time $\theta t_{i}^{T_{k}}$, and the communication latency $\theta l_{i}^{T_{k}}$, and the computation time $\omega_{i}^{T_{k}}$, respectively by:

$\theta t_{i}^{T_{k}}=\frac{\theta t_{i}^{T_{0} \times C_{k}}}{C_{0}}$

$\theta l_{i}^{T_{k}}=\frac{\theta l_{i}^{T_{0}} \times Z_{k}}{Z_{0}}$

$\omega_{i}^{T_{k}}=\theta t_{i}^{T_{k}}+\theta l_{i}^{T_{k}}$

- Computing the Node Performance index NPI ${ }^{\mathrm{Tk}}$

The TLBA agent gets the computed value of $\omega_{i}^{T_{k}}{ }_{\mathrm{i}}$, and computes the node performance index $\mathrm{NPI}_{\mathrm{i}}{ }^{\mathrm{T}} \mathrm{k}$ by:

$N P I_{i}^{T_{k}}=\frac{\operatorname{MIN}\left(\omega_{i}^{T_{k}}\right)}{\omega_{i}^{T_{k}}}$

- Computing the initial load $\mathrm{LB}^{\mathrm{Tk}}$

The TLBA agent gets the node performance index $N P I_{i}^{T_{k}}$, and computes the load assignment $\mathrm{LB}_{\mathrm{i}}$ by :

$L B_{i}^{T_{k}}=\mathrm{LB}_{\mathrm{ref}} \times \frac{N P I_{i}^{T_{k}}}{\left\langle N P I_{i}^{T_{k}}\right\rangle}$

Where :

$\left\langle\mathrm{NPI}_{\mathrm{i}}{ }_{\mathrm{k}}\right\rangle$ The average of node performance index $\mathrm{NPI}_{\mathrm{i}}$ that is computed by:

$\left\langle N P I_{i}^{T_{k}}\right\rangle=\frac{\sum_{i=0}^{n-1} N P I_{i}^{T_{k}}}{n}$

Step 3. Load Rebalancing

1) The TLBA agent computes the experimental computation time $\omega_{i}^{E X P}$ by:

$\omega_{i}^{E X P}(t)=\frac{\Omega_{i}^{E X P}(t)}{L B_{i}(t-1)}$

2) The TLBA agent computes the new performance index $N P I_{i}^{E X P}$ by:

$N P I_{i}^{E X P}=\frac{M I N\left(\omega_{i}^{E X P}(t)\right)}{\omega_{i}^{E X P}(t)}$

3) The TLBA agent computes the new load $\operatorname{LB}_{i}^{\mathrm{EXP}}(\mathrm{t})$ by:

$L B_{i}^{E X P}(t)=L B_{\text {ref }} \times \frac{N P I_{i}^{E X P}(t)}{\left\langle N P I_{i}^{E X P}(t)\right\rangle}$

4) The TLBA agent tests the overload $\triangle \mathrm{LB}_{\mathrm{i}}$ by:

$\Delta L B_{i}=L B_{i}^{E X P}(t)-L B_{i}^{E X P}(t-1)$

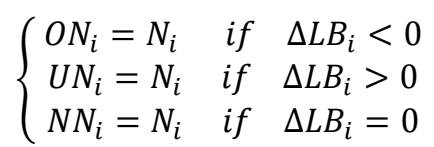

5) The TLBA agent determines the required load migration by running the given algorithm (Agent migration determination).

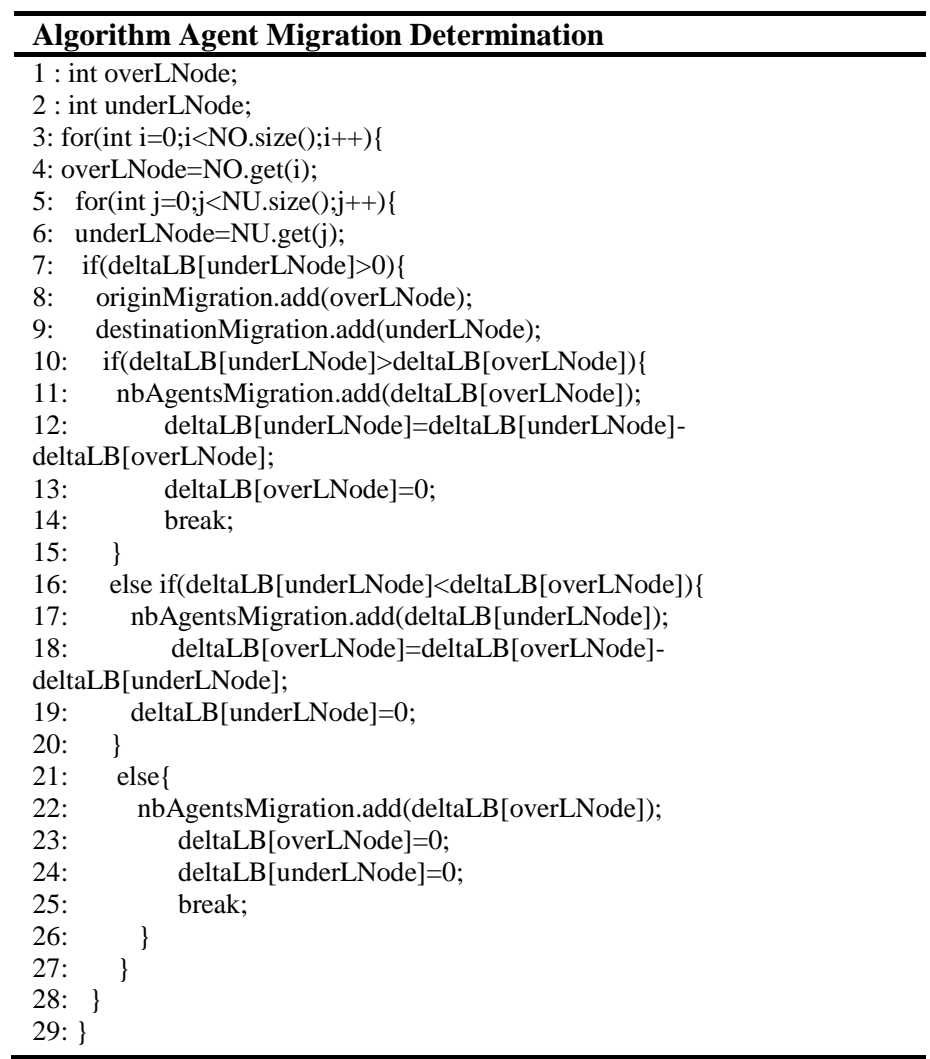

The load deltaLB(overLNode) (line 3) which corresponds to the overloaded node is compared with deltaLB(underLNode) of the under loaded node. This is done, to decide the required load migration with the node destination. When deltaLB(underLNode) is greater than deltaLB(overLNode) (line 10), the load will move to the under loaded node (line 11). At the end, this algorithm provides the three output results:

1) originMigration list of nodes from where the load will move.

2) destinationMigration list of nodes that will receive the load.

3) nbAgentsMigration list of agents load that will move from their origin node to the appropriate destination node.

\section{RESULTS AND DISCUSSION}

The proposed middleware is integrated in the parallel and distributed virtual machine [7], which is constituted by distributed computing system of 10 heterogeneous nodes. To do so, an SPMD application is chosen in order to perform the image processing of ne $\times$ me $=(20 \times 50)$ elementary images of size $(1024 \times 768)$ pixels. Thus, NA=1000 of AVPU(Agent Virtual Processing Units) agents have to execute the same task 
$\mathrm{T}_{\mathrm{k}}$ at the same time in the system. To illustrate the effectiveness feature of this middleware two case studies are considered:

\section{Case 1 Task assignment by initial performance test}

In this case the task assignment is performed by using the $\mathrm{LB}^{\mathrm{T} 0}$ in Table $\mathrm{I}$. The initial performance test is executed by using task $\mathrm{T}_{0}$ of complexity $\mathrm{C}_{0}(\mathrm{x})=\mathrm{O}\left(\mathrm{x}^{3}\right)$ and data $\mathrm{D}_{0}$ (matrix $(80 \times 80)$ where $\mathrm{x}_{0}=6400$ and $\left.\mathrm{y}_{0}=6401\right)$. For example in Table III, the value of $\Delta \mathrm{LB}_{8}$ at the node $\mathrm{N}_{8}$ is equal to -5 . This means that the node is overloaded by 5 agents, which have to move to under loaded nodes given in Table $\mathrm{V}$.

Case 2 Task assignment by prediction using the metadata $\mathrm{MDT}_{\mathrm{K}}$.
The node task assignment is performed by using the $\mathrm{LB}^{\mathrm{Tk}}$ in Table II. The predicted $\mathrm{LB}^{\mathrm{Tk}}$ is based on the metadata of task $\mathrm{T}_{\mathrm{k}}$ (complexity $\mathrm{C}_{\mathrm{k}}(\mathrm{x})=\mathrm{O}\left(\mathrm{x}^{2}\right)$, and data size $\left(\mathrm{x}_{\mathrm{k}}=786432\right.$, $\mathrm{y}_{\mathrm{k}}=786433$ ), and the metadata $\mathrm{MDT}_{0}$. In this case the load $\Delta \mathrm{LB}_{8}=\mathrm{LB}_{8}{ }^{\mathrm{EXP}(\mathrm{t})}-\mathrm{LB}_{8}{ }^{\mathrm{EXP}(\mathrm{t}-1)}$ of node $\mathrm{N}_{8}$ is equal to -1 in Table IV. This means that the node is overloaded by only one agent, which has to move to under loaded nodes given in Table VI.

By comparing the two cases (Fig. 3), the system is balanced from the first iteration in case 2 . Therefore, in case 1 it becomes balanced after the second iteration. This means that case 2 grants effective load balancing strategy of the system at the first iteration. The Fig. 4 presents that the system becomes balanced after performing the load rebalancing.

TABLE. I. Results of LOAD Assignment by Initial PERformance Test

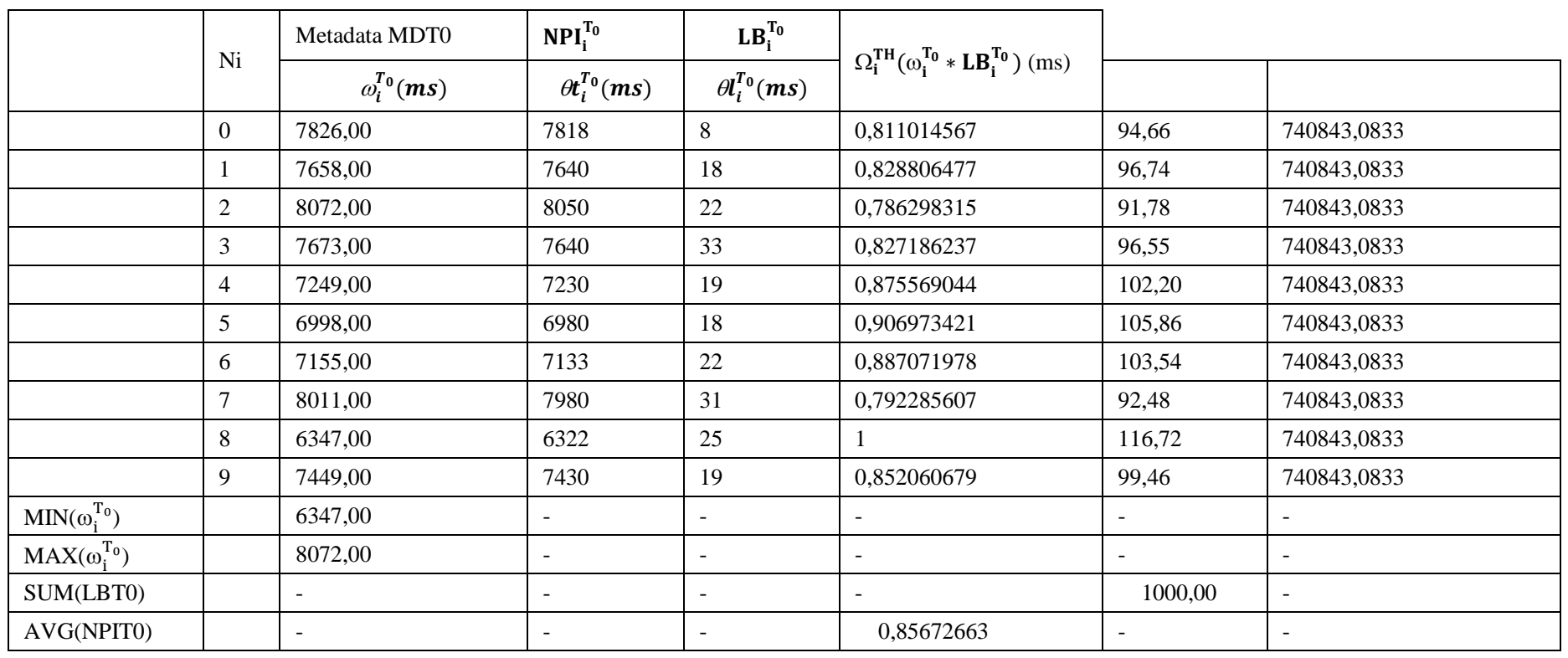

TABLE. II. RESUlts OF LOAD ASSIGNMENT BY PREDICTION

\begin{tabular}{|c|c|c|c|c|c|c|c|}
\hline & & Metadata MDTk & & & & & \\
\hline & NI & $\omega_{i}^{T_{k}}(m s)$ & $\theta l_{i}^{T_{k}}(\mathrm{~ms})$ & $\theta t_{i}^{T_{k}}(m s)$ & $\mathbf{N} \mathbf{P I}_{\mathbf{i}}$ & $\mathbf{L} \mathbf{b}_{i}$ & $\Omega_{i}$ \\
\hline & 0 & 19428,016 & 983,040 & 18444,976 & 0,925852089 & 103,22 & 2005359,82 \\
\hline & 1 & 20236,861 & 2211,840 & 18025,021 & 0,888846789 & 99,09 & 2005270,60 \\
\hline & 2 & 21695,693 & 2703,360 & 18992,333 & 0,829080199 & 92,43 & 2005332,89 \\
\hline & 3 & 22080,061 & 4055,040 & 18025,021 & 0,814647611 & 90,82 & 2005311,18 \\
\hline & 4 & 19392,430 & 2334,720 & 17057,710 & 0,927551072 & 103,41 & 2005371,19 \\
\hline & 5 & 18679,726 & 2211,840 & 16467,886 & 0,962940743 & 107,35 & 2005268,59 \\
\hline & 6 & 19532,218 & 2703,360 & 16828,858 & 0,92091277 & 102,67 & 2005372,86 \\
\hline & 7 & 22636,462 & 3809,280 & 18827,182 & 0,794623703 & 88,59 & 2005364,18 \\
\hline & 8 & 17987,469 & 3072,000 & 14915,469 & 1 & 111,48 & 2005243,08 \\
\hline & 9 & 19864,289 & 2334,720 & 17529,569 & 0,90551789 & 100,95 & 2005300,00 \\
\hline $\operatorname{MIN}\left(\omega_{i}^{T_{k}}\right)$ & & 17987,469 & - & - & - & - & - \\
\hline $\operatorname{MAX}\left(\omega_{i}^{T_{k}}\right)$ & & 22636,462 & - & - & - & - & - \\
\hline SUM(LBTk) & & - & - & - & - & 1000,00 & - \\
\hline AVG(NPITk) & & - & - & - & 0,89699729 & - & - \\
\hline
\end{tabular}


TABLE. III. RESULTS OF LOAD REBALANCING (CASE 1)

\begin{tabular}{|c|c|c|c|c|c|c|c|}
\hline $\mathrm{N}_{\mathrm{i}}$ & $\mathrm{LB}_{\mathrm{i}}^{\mathrm{EXP}}(\mathrm{t}-1)$ & $\Omega_{\mathrm{i}}^{\mathrm{EXP}}(\mathrm{t})(\mathrm{ms})$ & $\omega_{\mathrm{i}}^{\mathrm{EXP}}(\mathrm{t})(\mathrm{ms})$ & $\mathrm{NPI}_{\mathrm{i}}^{\mathrm{EXP}}(\mathrm{t})$ & $\mathrm{LB}_{\mathrm{i}}^{\mathrm{EXP}}(\mathrm{t})$ & $\Delta \mathrm{LB}_{\mathrm{i}}(\mathrm{t})$ & State \\
\hline 0 & 95 & 1845662 & 19428,01613 & 0,92585209 & 103 & 8,00 & Under \\
\hline 1 & 97 & 1962976 & 20236,86144 & 0,88884679 & 99 & 2,00 & Under \\
\hline 2 & 92 & 1996004 & 21695,6928 & 0,8290802 & 92 & 0,00 & Normal \\
\hline 3 & 97 & 2141766 & 22080,06144 & 0,81464761 & 91 & $-6,00$ & Over \\
\hline 4 & 101 & 1958635 & 19392,43008 & 0,92755107 & 103 & 2,00 & Under \\
\hline 5 & 106 & 1980051 & 18679,72608 & 0,96294074 & 107 & 1,00 & Under \\
\hline 6 & 104 & 2031351 & 19532,21837 & 0,92091277 & 103 & $-1,00$ & Over \\
\hline 7 & 94 & 2127827 & 22636,46208 & 0,7946237 & 90 & $-4,00$ & Over \\
\hline 8 & 116 & 2086546 & 17987,46931 & 1 & 111 & $-5,00$ & Over \\
\hline 9 & 98 & 1946700 & 19864,28928 & 0,90551789 & 101 & 3,00 & Under \\
\hline
\end{tabular}

TABLE. IV. REsults OF LOAD REBALANCING (CASE 2)

\begin{tabular}{|c|c|c|c|c|c|c|c|}
\hline $\mathrm{N}_{\mathrm{i}}$ & $\mathrm{LB}_{\mathrm{i}}^{\mathrm{EXP}}(\mathrm{t}-1)$ & $\Omega_{\mathrm{i}}^{\mathrm{EXP}}(\mathrm{t})(\mathrm{ms})$ & $\omega_{\mathrm{i}}^{\mathrm{EXP}}(\mathrm{t})(\mathrm{ms})$ & $N \mathrm{NPI}_{\mathrm{i}}^{\mathrm{EXP}}(\mathrm{t})$ & $\mathrm{LB}_{\mathrm{i}}^{\mathrm{EXP}}(\mathrm{t})$ & $\Delta \mathrm{LB}_{\mathrm{i}}(\mathrm{t})$ & State \\
\hline 0 & 103 & 2020514 & 19428,01613 & 0,92585209 & 104 & 1,00 & Under \\
\hline 1 & 99 & 2043923 & 20236,86144 & 0,88884679 & 101 & 2,00 & Under \\
\hline 2 & 92 & 1996004 & 21695,6928 & 0,8290802 & 92 & 0,00 & Normal \\
\hline 3 & 91 & 2009286 & 22080,06144 & 0,81464761 & 91 & 0,00 & Normal \\
\hline 4 & 103 & 1997420 & 19392,43008 & 0,92755107 & 103 & 0,00 & Normal \\
\hline 5 & 107 & 1961371 & 18679,72608 & 0,96294074 & 105 & $-2,00$ & Over \\
\hline 6 & 103 & 2011818 & 19532,21837 & 0,92091277 & 103 & 0,00 & Normal \\
\hline 7 & 90 & 2037282 & 22636,46208 & 0,7946237 & 90 & 0,00 & Normal \\
\hline 8 & 111 & 1978622 & 17987,46931 & 1 & 110 & $-1,00$ & Over \\
\hline 9 & 101 & 2006293 & 19864,28928 & 0,90551789 & 101 & 0,00 & Normal \\
\hline
\end{tabular}

TABLE. V. Results of AgEnt's Migration (CASE 1)

\begin{tabular}{|l|l|l|l|l|}
\hline & Origin Migration $\mathrm{N}_{\mathrm{i}}$ & Destination Migration $\mathrm{N}_{\mathrm{i}}$ & Nb Agents Migration & Load Migration Percentage \\
\hline & 3 & 0 & 6 & $37,50 \%$ \\
\hline SUM & - & - & 6 & $37,50 \%$ \\
\hline & 6 & 0 & - & 1 \\
\hline SUM & - & 0 & 1 & $6,25 \%$ \\
\hline & 7 & 1 & 1 & $6,25 \%$ \\
\hline & 7 & 4 & 2 & $6,25 \%$ \\
\hline & 7 & - & 1 & $12,50 \%$ \\
\hline SUM & - & 4 & 4 & $6,25 \%$ \\
\hline & 8 & 5 & 1 & $25,00 \%$ \\
\hline & 8 & 9 & 1 & $6,25 \%$ \\
\hline & 8 & - & 3 & $6,25 \%$ \\
\hline SUM & - & & 5 & $18,75 \%$ \\
\end{tabular}

TABLE. VI. RESUlts OF AgENT'S Migration (CASE 2)

\begin{tabular}{|l|l|l|l|l|}
\hline & $\begin{array}{l}\text { Origin Migration } \\
\mathrm{N}_{\mathrm{i}}\end{array}$ & Destination Migration $\mathrm{N}_{\mathrm{i}}$ & $\begin{array}{l}\text { Nb Agents } \\
\text { Migration }\end{array}$ & Load Migration Percentage \\
\hline & 5 & 0 & 1 & $33,33 \%$ \\
\hline & 5 & 1 & 1 & $33,33 \%$ \\
\hline SUM & - & - & 2 & $66,67 \%$ \\
\hline & 8 & 1 & 1 & $33,33 \%$ \\
\hline SUM & - & - & 1 & $33,33 \%$ \\
\hline
\end{tabular}




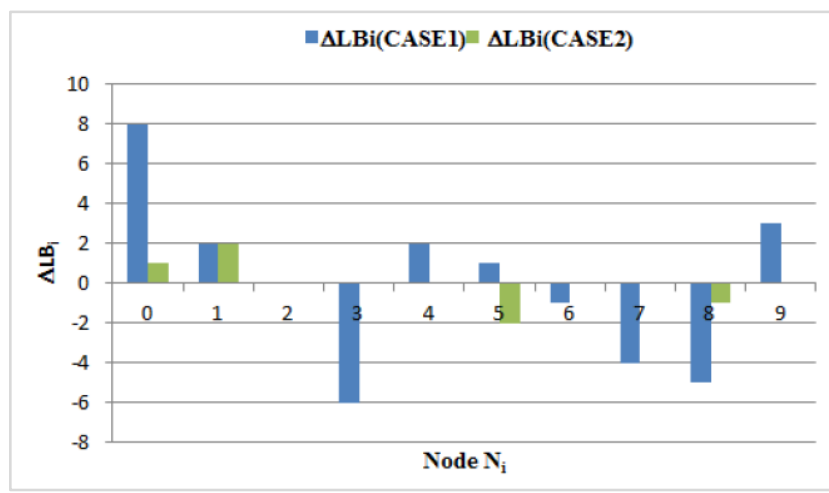

Fig. 3. Comparison of Overload in Case 1 and Case 2 of Each node $\mathrm{N}_{\mathrm{i} .}$

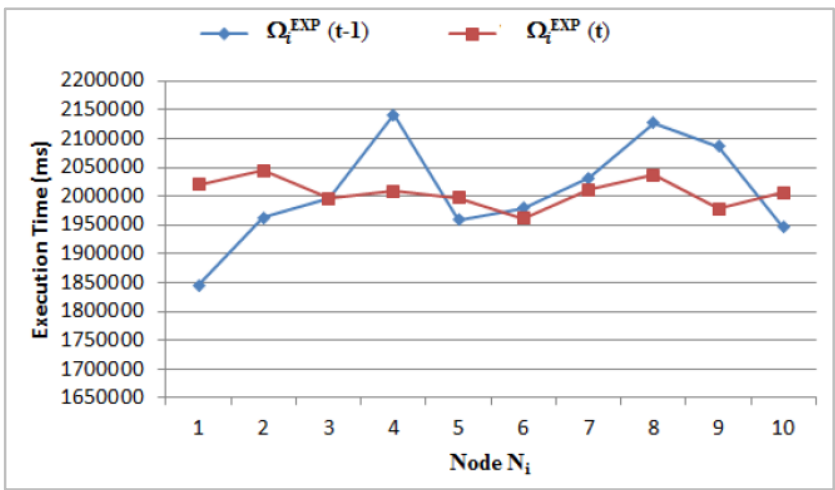

Fig. 4. Execution Time of the Task $T_{k}$ before and after Load Rebalancing.

By analyzing the execution time (Fig. 5) of both cases compared with the case 3 (unbalanced system where each node receives $L_{B}{ }_{i}^{\mathrm{NB}}=100$ agents), the following conclusions are achieved:

- In case 1 (Table VII) the obtained unbalance DES is $\operatorname{DES} 1=\Omega_{\mathrm{MAX}}^{\mathrm{EXP}}\left(\mathrm{LB}_{\mathrm{i}}^{\mathrm{T}_{0}}\right)-\Omega_{\mathrm{MIN}}^{\mathrm{EXP}}\left(\mathrm{LB}_{\mathrm{i}}^{\mathrm{T}_{0}}\right)=296,104 \mathrm{~s}$, and for case 2 is $\mathrm{DES} 2=\Omega_{\mathrm{MAX}}^{\mathrm{EXP}}\left(\mathrm{LB}_{\mathrm{i}}^{\mathrm{T}_{\mathrm{k}}}\right)-\Omega_{\mathrm{MIN}}^{\mathrm{EXP}}\left(\mathrm{LB}_{\mathrm{i}}^{\mathrm{T}_{\mathrm{k}}}\right)=$
41,2778496 s. These present the efficiency of task assignment based on prediction compared to the one based on initial performance test.

- The gain of performance $\varphi$ compared to case 3, provides to the following results:

- In case 2 the gain of performance is $\varphi 2=$ $\frac{\Omega_{M A X}^{E X P}\left(L B_{i}^{N B}\right)-\Omega_{M I N}^{E P P}\left(L B_{i}^{N B}\right)}{S_{M A X}\left(L B_{i}^{T_{L}}\right)-\Omega_{M X N}\left(L B_{i}^{T_{L}}\right)}=5,631$ at the first

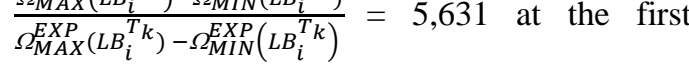
iteration.

- In case 1 the gain of performance is $\varphi 1=$ $\frac{\Omega_{M A X}^{E X P}\left(L B_{i}^{N B}\right)-\Omega_{M I N}^{E X P}\left(L B_{i}^{N B}\right)}{\Omega_{M A X}^{E P}\left(L B_{i}^{T_{0}}\right)-\Omega_{M I N}^{E P}\left(L B_{i}^{T_{0}}\right)}=1,570$ at the first iteration, which is enhanced to $\varphi^{\prime} 1=5,631$ after de second iteration.

- The obtained gain of performance of case $2 \varphi 2$ is equal to $\varphi^{\prime} 1$, which illustrates the effectiveness of the load rebalancing step based on load migration.

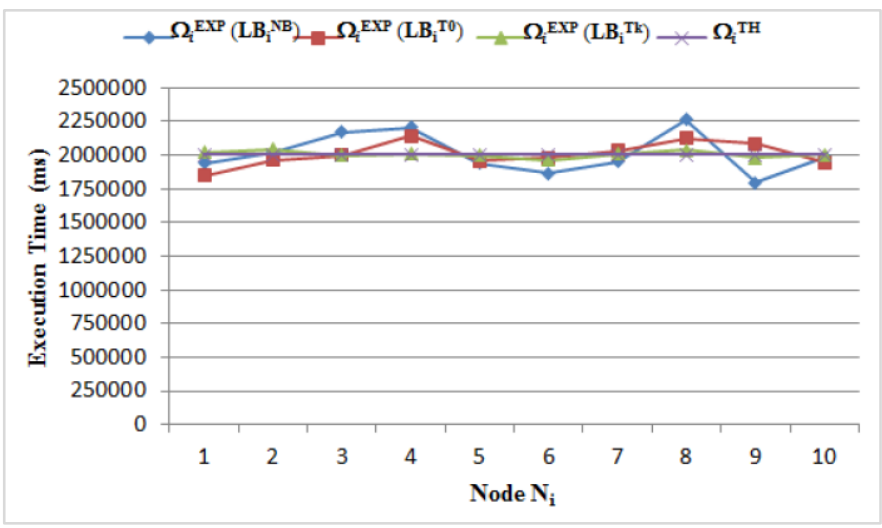

Fig. 5. Execution Time Comparison between Theoretical Execution Time $\Omega_{i}^{T H}$, and Experimental One of Case $1 \Omega_{i}^{E X P}\left(L B_{i}^{T_{0}}\right)$ and Case 2 $\Omega_{i}^{E X P}\left(L B_{i}^{T_{k}}\right)$ and Case $3 \Omega_{i}^{E X P}\left(L B_{i}^{T_{N B}}\right)$.

TABLE. VII. COMParison Based EXecution Time in Three CASES of LoAd Assignment

\begin{tabular}{|c|c|c|c|c|c|c|}
\hline \multirow{2}{*}{$\mathrm{N}_{\mathrm{i}}$} & \multirow{2}{*}{$\Omega_{\mathrm{i}}^{\mathrm{TH}}(\mathrm{ms})$} & \multicolumn{2}{|l|}{ CASE 1} & \multicolumn{2}{|l|}{ CASE 2} & \multirow{2}{*}{$\begin{array}{l}\text { CASE } 3 \\
\Omega_{\mathrm{i}}^{\mathrm{EXP}}\left(\mathrm{LB}_{\mathrm{i}}^{\mathrm{NB}}\right)(\mathrm{ms})\end{array}$} \\
\hline & & $\Omega_{\mathrm{i}}^{\mathrm{EXP}}\left(\mathrm{LB}_{\mathrm{i}}^{\mathrm{T}_{0}}\right)(\mathrm{ms})$ & $\varepsilon_{i}\left(\mathrm{LB}_{\mathrm{i}}^{\mathrm{T}_{0}}\right)$ & $\Omega_{\mathrm{i}}^{\mathrm{EXP}}\left(\mathrm{LB}_{\mathrm{i}}^{\mathrm{T}_{\mathrm{k}}}\right)(\mathrm{ms})$ & $\varepsilon_{i}\left(L_{i}^{T_{k}}\right)$ & \\
\hline 0 & 2005359,82 & 1845661,53 & 0,079635728 & 2020513,677 & 0,007556677 & 1942801,61 \\
\hline 1 & 2005270,60 & 1962975,56 & 0,021091936 & 2043923,005 & 0,019275406 & 2023686,14 \\
\hline 2 & 2005332,89 & 1996003,74 & 0,00465217 & 1996003,738 & 0,004652171 & 2169569,28 \\
\hline 3 & 2005311,18 & 2141765,96 & 0,068046686 & 2009285,591 & 0,001981942 & 2208006,14 \\
\hline 4 & 2005371,19 & 1958635,44 & 0,023305286 & 1997420,298 & 0,003964798 & 1939243,01 \\
\hline 5 & 2005268,59 & 1980050,96 & 0,012575687 & 1961371,238 & 0,021891009 & 1867972,61 \\
\hline 6 & 2005372,86 & 2031350,71 & 0,012954125 & 2011818,492 & 0,003214181 & 1953221,84 \\
\hline 7 & 2005364,18 & 2127827,44 & 0,061067841 & 2037281,587 & 0,015916015 & 2263646,21 \\
\hline 8 & 2005243,08 & 2086546,44 & 0,040545389 & 1978621,624 & 0,013275925 & 1798746,93 \\
\hline 9 & 2005300,00 & 1946700,35 & 0,029222386 & 2006293,217 & 0,000495296 & 1986428,93 \\
\hline
\end{tabular}




\section{RELATED WORK}

There are several inspiring load balancing approaches which have presented interesting results. Some of them are proposed for distributed systems [8], grid [9],[10], P2P systems [11],[12], and also for cloud computing systems [13],[14], and heterogeneous computing systems [15],[16],[17]. The main idea behind the considered challenge is the effective method for task assignment. To do so, the approaches in [18],[19] are based on the states of the nodes, which are grouped on domains. In [20], it is based on an index of load that is defined by the summation of the active services duration in the node; without taking into account the communication latency. The load prediction method is also investigated in load balancing models; such as [21] for dynamic load balancing of HLA based distributed simulations. However, system can further be unbalanced when running an application. This leads authors to propose dynamic load balancing algorithm based-load migration [22] in order to move the load from the overloaded nodes to the under loaded ones. It is performed by moving just one unit per iteration [23],[24],[25],[26], or a fixed number [27],[28]. Further, in [29] it's achieved by exchanging the load between neighbor nodes.

Multi-agent based load-balancing approach has been investigated as a promising paradigm for this challenge. The agent is used in [30] to represents the node for load balancing process, and in [31] it is implemented to monitor and detect the congestion in the nodes, and [32] to perform the load assignment. Further, in [33] the agent is deployed to encapsulate the tasks that will be executed.

Thereby, the mobile agents allow the load migration between nodes even in [34] homogeneous distributed system, or heterogeneous one based on node prediction algorithm. Accordingly, the proposed middleware combines two methods; task assignment according to node performance, and task migration, by the way to assign a set of tasks $T_{k}\{k=1, \ldots, N T\}$ to a set of $\mathrm{N}_{\mathrm{i}}\{\mathrm{i}=1, \ldots, \mathrm{n}\}$ nodes. Through, thanks to these several interesting works, the proposed work develops their foundation in the following ways. In this paper, a new load balancing middleware for distributed computing systems is proposed and implemented with three main focuses:

- Effective task assignment method using node performance prediction based on communication latency of each node, with integrated task migration algorithm.

- Optimized load balancing time by using the asynchronous communication mechanism between agents.

- Scalable Load balancing middleware for SPMD applications based parallel and distributed computing systems.

\section{VII.CONCLUSION}

The proposed load balancing middleware based cooperative mobile agents team work is a new paradigm, which is implemented using the aspect oriented approach for separating the load balancing aspect from distributed system. Through, this middleware can be integrated with different distributed computing systems for node task assignment problem. The proposed middleware deploys the mobile agent for each node in order to get the node performance. When the program is deployed the mobile agents perform an initial performance test based on referenced task in order to compute the appropriate task assignment of each node. In the case when the application tasks metadata; the complexity and the data size are known, the proposed method can predict with precision the task assignment for each node. When the application is running, if the system becomes unbalanced, the middleware executes the rebalancing algorithm to identify and decide the required load migration and rebalance the system. The obtained results, related to the execution time of each node and the gain of performance, demonstrates that the proposed middleware can ensure effective balanced distributed computing system and enhance its performance. Further, it is interesting to have a load balancing solution which handles the middleware failures. To do so, an extended work is driven in order to propose and implement a fault tolerance module for the proposed load balancing middleware.

\section{REFERENCES}

[1] M. D. Assunção, R. N. Calheiros, S. Bianchi, M. A. S. Netto, and R. Buyya, "Big Data computing and clouds: Trends and future directions," J. Parallel Distrib. Comput., vol. 79, pp. 3-15, 2015.

[2] A. Puder, K. Romer, and F. Pilhofer, "Distributed Systems Architecture,” Morgan Kaufmann Publ., vol. 53, no. 9, pp. 1689-1699, 2006.

[3] W. R. Braun, P., \& Rossak, Mobile agents: Basic concepts, mobility models, and the tracy toolkit. 2005.

[4] F. Bellifemine, G. Caire, and D. Greenwood, Developing Multi-Agent Systems with JADE. 2007.

[5] E. Hilsdale and J. Hugunin, "Advice Weaving in AspectJ," in Proceedings of the 3rd International Conference on Aspect-oriented Software Development, 2004, pp. 26-35.

[6] M. Lippert and C. V. Lopes, "A Study on Exception Detection and Handling Using Aspect-oriented Programming," in Proceedings of the 22Nd International Conference on Software Engineering, 2000, pp. 418427.

[7] F. Z. Benchara, M. Youssfi, O. Bouattane, H. Ouajji, and M. O. Bensalah, "A New Distributed Computing Environment Based on Mobile Agents for SPMD Applications BT-Proceedings of the Mediterranean Conference on Information \& Communication Technologies 2015," 2016, pp. 353-362.

[8] Y. Jiang, "A Survey of Task Allocation and Load Balancing in Distributed Systems," IEEE Trans. Parallel Distrib. Syst., vol. 27, no. 2, pp. 585-599, 2016.

[9] D. S. Acker and S. Kulkarni, "A dynamic load dispersion algorithm for load-balancing in a heterogeneous grid system," in 2007 IEEE Sarnoff Symposium, 2007, pp. 1-5.

[10] M. Li, P. He, and L. Zhao, "Dynamic Load Balancing Applying WaterFilling Approach in Smart Grid Systems," IEEE Internet of Things Journal, vol. 4, no. 1. pp. 247-257, 2017.

[11] D. R. Karger and M. Ruhl, "Simple Efficient Load Balancing Algorithms for Peer-to-peer Systems," in Proceedings of the Sixteenth Annual ACM Symposium on Parallelism in Algorithms and Architectures, 2004, pp. 36-43.

[12] R. Bhardwaj, V. S. Dixit, and A. K. Upadhyay, "A propound method for agent based dynamic load balancing algorithm for heterogeneous P2P systems," 2009 International Conference on Intelligent Agent \& MultiAgent Systems. pp. 1-4, 2009.

[13] H. C. Hsiao, H. Y. Chung, H. Shen, and Y. C. Chao, "Load Rebalancing for Distributed File Systems in Clouds," IEEE Trans. Parallel Distrib. Syst., vol. 24, no. 5, pp. 951-962, 2013.

[14] J. Zhao, K. Yang, X. Wei, Y. Ding, L. Hu, and G. Xu, "A Heuristic Clustering-Based Task Deployment Approach for Load Balancing Using 
Bayes Theorem in Cloud Environment," IEEE Transactions on Parallel and Distributed Systems, vol. 27, no. 2. pp. 305-316, 2016.

[15] J. Shen, A. L. Varbanescu, Y. Lu, P. Zou, and H. Sips, "Workload Partitioning for Accelerating Applications on Heterogeneous Platforms," IEEE Transactions on Parallel and Distributed Systems, vol. 27, no. 9. pp. 2766-2780, 2016.

[16] E. Hwang, S. Kim, T. k. Yoo, J. S. Kim, S. Hwang, and Y. r. Choi, "Resource Allocation Policies for Loosely Coupled Applications in Heterogeneous Computing Systems," IEEE Transactions on Parallel and Distributed Systems, vol. 27, no. 8. pp. 2349-2362, 2016.

[17] J. Y. Jang, H. Wang, E. Kwon, J. W. Lee, and N. S. Kim, "WorkloadAware Optimal Power Allocation on Single-Chip Heterogeneous Processors," IEEE Transactions on Parallel and Distributed Systems, vol. 27, no. 6. pp. 1838-1851, 2016.

[18] F. auf der Meyer Heide, B. Oesterdiekhoff, and R. Wanka, "Strongly adaptive token distribution," Algorithmica, vol. 15, no. 5, pp. 413-427, 1996.

[19] P. Berenbrink, T. Friedetzky, and R. Martin, "Dynamic Diffusion Load Balancing BT - Automata, Languages and Programming: 32nd International Colloquium, ICALP 2005, Lisbon, Portugal, July 11-15, 2005. Proceedings," L. Caires, G. F. Italiano, L. Monteiro, C. Palamidessi, and M. Yung, Eds. Berlin, Heidelberg: Springer Berlin Heidelberg, 2005, pp. 1386-1398.

[20] Y. Qiao and G. v. Bochmann, "A Diffusive Load Balancing Scheme for Clustered Peer-to-Peer Systems," 2009 15th International Conference on Parallel and Distributed Systems. pp. 842-847, 2009.

[21] R. E. De Grande, A. Boukerche, and R. Alkharboush, "Time SeriesOriented Load Prediction Model and Migration Policies for Distributed Simulation Systems," IEEE Transactions on Parallel and Distributed Systems, vol. 28, no. 1. pp. 215-229, 2017.

[22] G. Cybenko, "Dynamic load balancing for distributed memory multiprocessors," J. Parallel Distrib. Comput., vol. 7, no. 2, pp. 279301, 1989.

[23] A. Cortés, A. Ripoll, F. Cedó, M. A. Senar, and E. Luque, "An asynchronous and iterative load balancing algorithm for discrete load model," J. Parallel Distrib. Comput., vol. 62, no. 12, pp. 1729-1746, 2002.
[24] Y. F. Hu and R. J. Blake, "An improved diffusion algorithm for dynamic load balancing," Parallel Comput., vol. 25, no. 4, pp. 417-444, 1999.

[25] E. Luque, A. Ripoll, A. Cortes, and T. Margalef, "A distributed diffusion method for dynamic load balancing on parallel computers," Proceedings Euromicro Workshop on Parallel and Distributed Processing. pp. 43-50, 1995.

[26] T. A. Murphy and J. G. Vaughan, "On the relative performance of diffusion and dimension exchange load balancing in hypercubes," in PDP, 1997.

[27] P. Berenbrink, T. Friedetzky, and Z. Hu, "A new analytical method for parallel, diffusion-type load balancing," J. Parallel Distrib. Comput., vol. 69, no. 1, pp. 54-61, 2009.

[28] F. Cedo, A. Cortes, A. Ripoll, M. A. Senar, and E. Luque, "The Convergence of Realistic Distributed Load-Balancing Algorithms," Theory Comput. Syst., vol. 41, no. 4, pp. 609-618, 2007.

[29] I. Konstantinou, D. Tsoumakos, and N. Koziris, "Fast and CostEffective Online Load-Balancing in Distributed Range-Queriable Systems," IEEE Transactions on Parallel and Distributed Systems, vol. 22, no. 8. pp. 1350-1364, 2011.

[30] S. Banerjee and J. P. Hecker, "A Multi-agent System Approach to LoadBalancing and Resource Allocation for Distributed Computing BT First Complex Systems Digital Campus World E-Conference 2015," P. Bourgine, P. Collet, and P. Parrend, Eds. Cham: Springer International Publishing, 2017, pp. 41-54.

[31] X.-J. Shen et al., "Achieving dynamic load balancing through mobile agents in small world P2P networks," Comput. Networks, vol. 75, pp. 134-148, 2014.

[32] O. Rihawi, Y. Secq, and P. Mathieu, "Load-Balancing for Large Scale Situated Agent-based Simulations," Procedia Comput. Sci., vol. 51, pp. 90-99, 2015.

[33] S. Hunt, Q. Meng, C. Hinde, and T. Huang, "A Consensus-Based Grouping Algorithm for Multi-agent Cooperative Task Allocation with Complex Requirements," Cognit. Comput., vol. 6, no. 3, pp. 338-350, Sep. 2014.

[34] J. Liu, X. Jin, and Y. Wang, "Agent-based load balancing on homogeneous minigrids: macroscopic modeling and characterization," IEEE Trans. Parallel Distrib. Syst., vol. 16, no. 7, pp. 586-598, 2005. 